\title{
Dynamic Channel Assignment in IEEE 802.11 Networks
}

\author{
Robert Akl \\ Dept of Computer Science and Eng. \\ University of North Texas \\ Denton, Texas, 76207 \\ rakl@cse.unt.edu
}

\author{
Anurag Arepally \\ Dept of Computer Science and Eng. \\ University of North Texas \\ Denton, Texas, 76207 \\ aa0118@cse.unt.edu
}

\begin{abstract}
We design a dynamic channel assignment algorithm for IEEE 802.11 wireless networks. Our algorithm assigns channels dynamically in a way that minimizes channel interference generated by neighboring access points (APs) on a reference access point, resulting in higher throughput. We implement and simulate our algorithm using two versions (I: pick rand and II: pick first) and different number of $\operatorname{APs}(4,9,16$, and 25). Analysis of our algorithm shows an improvement by a factor of 4 (by lowering the total interference on an AP by $6 \mathrm{dBm}$ on average) over default settings of having all APs use the same channel. As the number of APs is increased in a given service area, dynamic channel assignment becomes crucial; otherwise overlapping channel interference becomes a limiting factor.
\end{abstract}

\section{INTRODUCTION}

Tremendous growth and wide usage of IEEE 802.11 with increasing user access has raised issues like quality of service, channel interference, and network load management. The increase in deployment of access points (APs) leads to co-channel interference from neighboring APs degrading the network throughput. Issues related to resource management and interference impede the performance of WLANs.

Frequency assignment [1] is a major problem in designing wireless networks. All APs share the same frequency, which leads to interference that should be minimized and avoided if necessary, using efficient assignment of channels. In [1], the authors formulate an integer linear programming problem to optimize the channel assignment for hot spot service areas. The objective of their optimization is to minimize the maximum channel utilization, yielding higher throughput. In [2], the authors discuss the implementation of IEEE 802.11 based WLANs for enterprise customers with limitations like performance under heavy load, deployment issues, and network management. The authors suggest using dynamic resource management over static radio resource management to improve performance of large scale WLANs. Analysis of channel assignment for $802.11 \mathrm{~b}$ using radio interference is done in [3]. Experimental results show that performance differs with changing environmental conditions and channel separation when using different models. Also, a frequency separation of 3 or 4 channels between APs is suitable. In [4], the authors assign frequencies to APs using an algorithm that has an exponential computational complexity. To overcome the computationally intensive algorithm, the authors also use a greedy algorithm that is close to optimal but may not yield the optimal frequency assignment for a given WLAN. In [5], the authors propose techniques to improve the usage of wireless spectrum in WLANs. The authors emphasize that the use of a least congested channel search for assigning new channels for interfering APs is not efficient with continued growth of WLANs.

Our contributions are two-folds. First, we develop a mathematical model that captures the amount of interference between overlapping channels for IEEE 802.11 WLAN systems. Second, we design a dynamic channel allocation algorithm by minimizing channel interference between APs. Assigning optimal channels to APs is a crucial process for overall performance. Our algorithm assigns channels in a way that minimizes overlapping channel interference resulting in higher throughput. Numerical results are presented and analyzed for 4, 9, 16, and 25 APs.

The remainder of this paper is organized as follows. Channel interference in 802.11 is presented in Section II. In Section III, we define our overlapping channel interference factor. Our dynamic channel assignment algorithm is described in Section IV. Numerical results are presented in Section V, and finally Section VI concludes the paper.

\section{OVERLAPPING CHANNEL INTERFERENCE}

802.11b/g networks operate between $2.4 \mathrm{GHz}$ and $2.5 \mathrm{GHz}$ [6], [7]. In $802.11 \mathrm{~b} / \mathrm{g}$, transmissions between APs and demand clusters do not use a single frequency. Instead, the frequencies are divided into 14 channels, and use a modulation technique, direct sequence spread spectrum, to spread the transmission over multiple channels for effective uses of the frequency spectrum. Channel 1 is assigned to $2.412 \mathrm{GHz}$. There is 5 
$\mathrm{MHz}$ separation between the channels. Thus, channel 14 is assigned to $2.484 \mathrm{GHz}$. In the United States, channels 1-11 are used. Europe uses channels 1-13. Japan uses channels 1-14. An $802.11 \mathrm{~b} / \mathrm{g}$ signal occupies approximately $30 \mathrm{MHz}$ of the frequency spectrum. As a result, an $802.11 \mathrm{~b} / \mathrm{g}$ signal overlaps with several adjacent channel frequencies.

Channels should be assigned to APs such that overlapping channel interference is minimized. Channels are reused because of limited availability. The same channel should be assigned to two APs, which are located far enough apart, if the overlapping channel interference signal detected by each AP is less than a given threshold.

Use of overlapping channels degrades network throughput. Interference in $802.11 \mathrm{~b}$ causes APs and stations to send frames over and over again to increase the odds of successful transmission. Typically, if devices were to send one copy of a frame, data is transmitted at $11 \mathrm{Mbps}$ (54 Mbps for $802.11 \mathrm{~g})$. However, if the efficiency were to drop to $50 \%$, for instance, because of interference, the devices would still be transmitting at $11 \mathrm{Mbps}$, but it would be duplicating each frame, making the effective throughput 5.5 Mbps. Therefore, 802.11 networks will have a significant decrease in network performance because of interference.

\section{OVERlapping CHANNEL INTERFERENCE FACTOR}

We model overlapping channel interference by defining an overlapping channel interference factor, $w_{i j}$, to be the relative percentage increase in interference as a result of two APs $i$ and $j$ using overlapping channels. Thus overlapping channels assigned to APs must be chosen carefully.

The overlapping channel interference factor is defined as:

$$
w_{i j}= \begin{cases}1-\left|F_{i}-F_{j}\right| \times c & \text { if } w_{i j} \geq 0 \\ 0 & \text { otherwise }\end{cases}
$$

where $F_{i}$ is the channel assigned to AP $i . F_{i}$ belongs to the set of available channels. $c$ is the overlapping channel factor. In $802.11 \mathrm{~b} / \mathrm{g}, c$ is $1 / 5$ where 5 is the maximum number of overlapping channels.

For instance, if channel 1 is assigned to AP $i$ and channel 1 is also assigned to $\mathrm{AP} j$, the overlapping channel interference factor between $\mathrm{AP} i$ and $\mathrm{AP} j, w_{i j}$, is 1.0 or $100 \%$. If channel 5 is assigned to $\mathrm{AP} j, w_{i j}$ is 0.2 or $20 \%$. If channels 6 or higher is assigned to $\mathrm{AP} j, w_{i j}$ is 0 or $0 \%$, which means there is no interference between $\mathrm{AP} i$ and $\operatorname{AP} j$.

\section{Dynamic Channel Assignment Algorithm}

We present a dynamic channel assignment algorithm for IEEE 802.11 WLAN systems. Channels should be assigned to each AP in such a way that minimizes interference between APs. The closer the overlapping frequencies, the higher the interference.
We formulate our dynamic channel assignment problem using the following variables defined as follows:

- $\mathbf{A}_{\mathbf{i}}$ is the set of neighboring APs to AP $i$.

- $c$ is the overlapping channel factor.

- $d_{i j}$ is the distance between AP $i$ and AP $j$.

- $F_{i}$ is the channel assigned to AP $i$.

- $I_{i j}$ is the interference that AP $j$ causes on AP $i$.

- $K$ is the total number of available channels. $802.11 \mathrm{~b} / \mathrm{g}$ has 11 channels.

- Loss is a function that captures the attenuation loss based on the propagation model used.

- $m$ is a pathloss exponent.

- $P_{i}$ is the transmit power of AP $i$.

- $Q_{i}$ is the cardinality of $\mathbf{A}_{\mathbf{i}}$.

- $w_{i j}$ is the overlapping channel interference factor between AP $i$ and AP $j$.

The dynamic channel assignment problem is given as:

$$
\min _{F_{i}} \sum_{j=1}^{Q_{i}} I_{i j}
$$

subject to

$$
\begin{aligned}
& I_{i j}=\frac{w_{i j} P_{j}}{\operatorname{Loss}\left(d_{i j}, m\right)}, \quad(2.2) \\
& w_{i j}= \begin{cases}1-\left|F_{i}-F_{j}\right| \times c & \text { if } w_{i j} \geq 0, \\
0 & \text { otherwise, }\end{cases}
\end{aligned}
$$

for $j \in \mathbf{A}_{\mathbf{i}}$,

for $F_{i} \in\{1, \ldots, K\}$.

Objective (2.1) minimizes the maximum total interference at each AP. Constraint (2.2) defines the interference between AP $i$ and AP $j$. Constraint (2.3) defines the overlapping channel interference factor between $\mathrm{AP} i$ and $\mathrm{AP} j$, which have been assigned channels $F_{i}$ and $F_{j}$, respectively.

Each AP would periodically (or when the amount of interference is above a threshold) run the above given dynamic channel assignment problem. Each AP would in turn pick its own channel that would minimize that amount of interference it receives from its neighbors. Of course as one AP changes its own channel, it would impact the interference on the APs of which it is a neighbor. Assuming that an AP would have on average six neighboring APs and since the number of total possible channels is eleven, the dynamic channel assignment can run within a few seconds, even if every possible channel is enumerated.

Analysis of our algorithm is carried out using two versions developed for breaking ties for channel assignment. We named these algorithm I (pick rand) and algorithm II (pick first). In algorithm I, we randomly break ties between channels that yield the same performance and randomly select a channel for assignment. For example if our algorithm selects 7, 8, 9, 10, 
and 11 as possible channels for assignment then the algorithm randomly picks say 9 in one instance. Algorithm II picks the first (smallest) channel number from channels that yield the same performance and assigns that to the AP. For example if our algorithm selects $7,8,9,10$, and 11 as possible channels then algorithm II assigns 7 every time.

The questions of the existence and uniqueness of the solution and whether the iterative approach in fact converges to the solution (if a unique solution exists) are generally difficult to answer due to the complexity of the equations involved. Kelly has shown that, for fixed alternate routing, the solution to the fixed point problem is in fact not unique [8]; in all the numerical examples we solved, the iterative approach converged to a unique solution in under 50 iterations.

\section{Numerical Results}

The simulations were carried out with service areas consisting of 4, 9, 16, and 25 APs forming a wireless LAN. Initially, we assign default factory settings, i.e., all APs are assigned the same channel number, and calculate the total interference caused on each AP from other APs in the network. These results are used as a benchmark to compare with our dynamic channel assignment algorithm.

The simulations were performed using the design procedures specified in [9]. APs are placed 150 meters apart from each other. We assume the following for the analysis.The transmit power of individual APs is set to $20 \mathrm{dBm}$. The receiver sensitivity threshold is $-84 \mathrm{dBm}$. The pathloss exponent is 2 .

Next, we assign the same channel for all APs starting with Channel 1 and simulate our algorithm for 100 runs. This is repeated for Channels 2 through 11. We compare our results for total interference from algorithms I and II with default factory setting for 4, 9, 16, and 25 APs.

\section{A. Dynamic Channel Assignment for WLAN with 4 APs}

Initially, we place 4 APs to simulate the WLAN. Setting initially all APs to the same channels, we simulate our dynamic channel assignment algorithm for 100 runs while recording the interference and channel assignment from each run. We calculate the total interference in $\mathrm{dBm}$ for algorithm I and II and compare with benchmark settings. The total interference ranges between $-34 \mathrm{dBm}$ to $-26 \mathrm{dBm}$ for both algorithm I and II while if all APs were still assigned the same channels, it is at $-19.5 \mathrm{dBm}$. Fig. 1 shows the results of total interference in $\mathrm{dBm}$ for Algorithm I and II compared to same channel assignment.

Table I shows the channel assignment map for WLAN with 4 APs after running the dynamic channel assignment algorithm for 100 runs and the interference values in $\mathrm{dBm}$ for the individual APs after dynamic channel assignment. The algorithm converged to a unique solution in under 10 iterations on average.

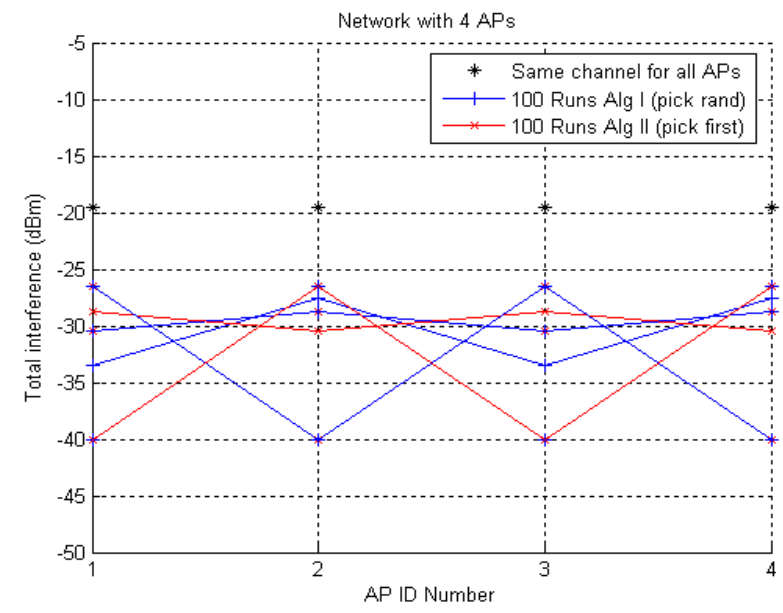

Fig. 1. Total Interference for AP 4 using algorithm I (pick rand) and II (pick first) comparing with same channel assignment. The different lines correspond to different initial conditions (i.e., initial AP channel numbers).

TABLE I

INTERFERENCE CALCULATED AT THE APS AFTER RUNNING DYNAMIC CHANNEL ASSIGNMENT ALGORITHM FOR WLAN WITH 4 APS.

\begin{tabular}{|c|c|c|}
\hline AP ID & CHANNEL $F_{i}$ & INTERFERENCE $(\mathrm{dBm})$ \\
\hline \hline$A P_{1}$ & 11 & -30.5115 \\
\hline$A P_{2}$ & 3 & -28.7506 \\
\hline$A P_{3}$ & 8 & -30.5115 \\
\hline$A P_{4}$ & 1 & -28.7506 \\
\hline \hline
\end{tabular}

\section{B. Dynamic Channel Assignment for WLAN with 9 APs}

Next, we simulate for 9 APs. Following the same procedure described in $\mathrm{V}-\mathrm{A}$, we generate our results and calculate the total interference. Fig. 2 shows the total interference from algorithm I and II compared with same channel assignment. The total interference for algorithm I and II varies from -28 $\mathrm{dBm}$ to $-20.5 \mathrm{dBm}$ while for the same channel assignment, it varies from $-18 \mathrm{dBm}$ to $-15.5 \mathrm{dBm}$. Observe that $\mathrm{AP} 3$ has the highest interference compared to other APs as it is at the center as seen in Fig 5 (ignore APs 10-25). Also, with the increase in the number of APs in WLAN there is an increase in the total interference. Our results show an improvement of $6 \mathrm{dBm}$ or 4 times that of the total interference from default factory settings. The algorithm converged to a unique solution in under 15 iterations on average.

Table II shows the channel assignment map for WLAN with 9 APs after running the dynamic channel assignment algorithm and the interference values for the corresponding channel numbers from the channel assignment map.

\section{Dynamic Channel Assignment for WLAN with 16 APs}

Next, we simulate 16 APs. Fig. 3 shows the total interference from algorithm I (pick rand) and II (pick first) compared with same channel assignment. The total interference for 


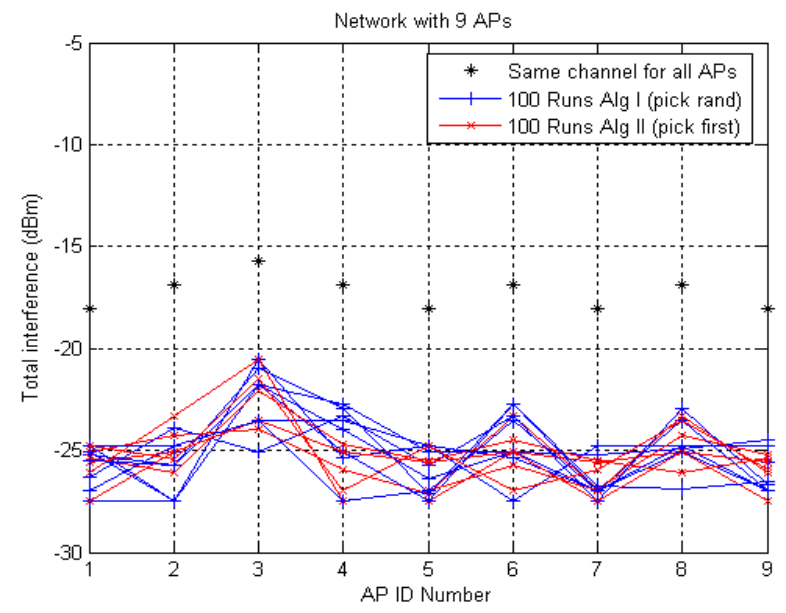

Fig. 2. Total Interference for AP 9 using algorithm I (pick rand) and II (pick first) comparing with same channel assignment. The different lines correspond to different initial conditions (i.e., initial AP channel numbers).

TABLE II

INTERFERENCE CALCULATED AT THE APS AFTER RUNNING DYNAMIC CHANNEL ASSIGNMENT ALGORITHM FOR WLAN WITH 9 APS.

\begin{tabular}{|c|c|c|}
\hline AP ID & CHANNEL $F_{i}$ & INTERFERENCE $(\mathrm{dBm})$ \\
\hline \hline$A P_{1}$ & 4 & -26.3202 \\
\hline$A P_{2}$ & 9 & -23.9314 \\
\hline$A P_{3}$ & 1 & -25.0708 \\
\hline$A P_{4}$ & 11 & -23.3099 \\
\hline$A P_{5}$ & 1 & -25.7403 \\
\hline$A P_{6}$ & 11 & -23.3099 \\
\hline$A P_{7}$ & 6 & -27.4473 \\
\hline$A P_{8}$ & 11 & -22.9148 \\
\hline$A P_{9}$ & 6 & -26.7094 \\
\hline \hline
\end{tabular}

algorithm I and II varies from $-27 \mathrm{dBm}$ to $-20 \mathrm{dBm}$ while for the same channel assignment, it varies from $-18 \mathrm{dBm}$ to -15 $\mathrm{dBm}$. Observe that APs 3, 6, 7, and 8 have high interference compared to other APs due to their placement as seen in Fig 5 (ignore APs 17-25). Our results show an improvement of a factor of 4 over default factory settings leading to higher throughput of the entire network. The algorithm converged to a unique solution in under 25 iterations on average.

The channel assignment map for WLAN with 16 APs after running the dynamic channel assignment algorithm and the corresponding interference values $(\mathrm{dBm})$ are shown in Table III.

\section{Dynamic Channel Assignment for WLAN with 25 APs}

Finally, we simulate 25 APs. Fig. 4 shows total interference from algorithm I (pick rand) and II (pick first) compared to same channel assignment. The total interference from algorithm I and II varies from $-25 \mathrm{dBm}$ to $-19 \mathrm{dBm}$ while for the same channel assignment, it varies from $-17 \mathrm{dBm}$ to -14 $\mathrm{dBm}$. With the increase in the number of APs in the network, we observe an increase in the total interference generated at

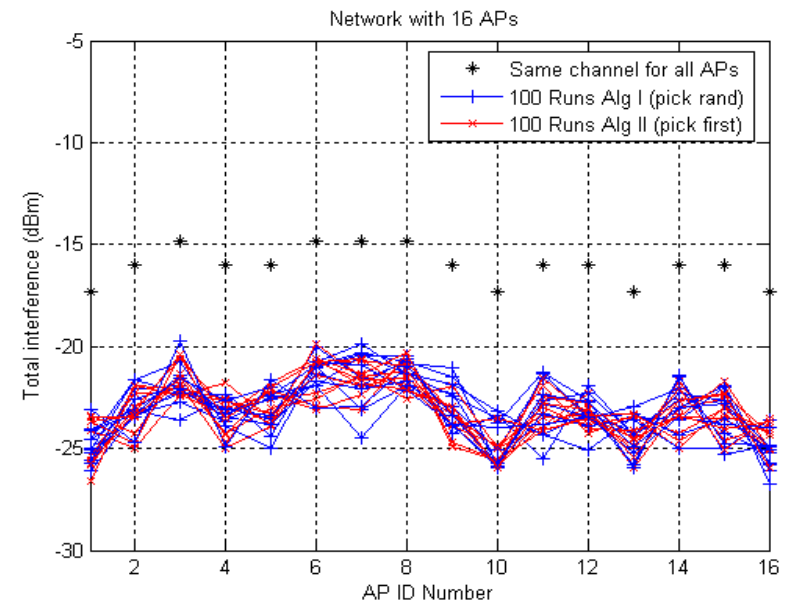

Fig. 3. Total Interference for AP 16 using algorithm I (pick rand) and II (pick first) comparing with same channel assignment. The different lines correspond to different initial conditions (i.e., initial AP channel numbers).

TABLE III

INTERFERENCE CALCULATED AT THE APS AFTER RUNNING DYNAMIC CHANNEL ASSIGNMENT ALGORITHM FOR WLAN WITH 16 APS.

\begin{tabular}{|c|c|c|}
\hline AP ID & CHANNEL $F_{i}$ & INTERFERENCE $(\mathrm{dBm})$ \\
\hline \hline$A P_{1}$ & 1 & -23.6595 \\
\hline$A P_{2}$ & 6 & -23.3692 \\
\hline$A P_{3}$ & 1 & -21.8192 \\
\hline$A P_{4}$ & 11 & -24.9920 \\
\hline$A P_{5}$ & 11 & -23.9314 \\
\hline$A P_{6}$ & 6 & -20.7229 \\
\hline$A P_{7}$ & 11 & -2185 \\
\hline$A P_{8}$ & 6 & -21.5906 \\
\hline$A P_{9}$ & 1 & -23.2224 \\
\hline$A P_{10}$ & 1 & -25.8278 \\
\hline$A P_{11}$ & 9 & -21.5286 \\
\hline$A P_{12}$ & 4 & -23.3506 \\
\hline$A P_{13}$ & 9 & -23.3458 \\
\hline$A P_{14}$ & 1 & -24.6180 \\
\hline$A P_{15}$ & 11 & -23.4146 \\
\hline$A P_{16}$ & 6 & -24.1758 \\
\hline \hline
\end{tabular}

the APs in the WLAN. The algorithm converged to a unique solution in under 50 iterations on average.

Fig. 5 shows a channel assignment map for WLAN with 25 APs after running the dynamic channel assignment algorithm. Table IV shows the corresponding interference values for the APs.

\section{Conclusions}

We develop a mathematical model that captures the amount of interference between overlapping channels for IEEE 802.11 WLAN systems. This is used in the design of a dynamic channel assignment algorithm. The dynamic channel assignment algorithm uses the channel information from neighboring APs in the network while selecting one of the APs as a reference AP. Our algorithm minimizes the interference among the APs and improves the throughput of the whole network. We imple- 


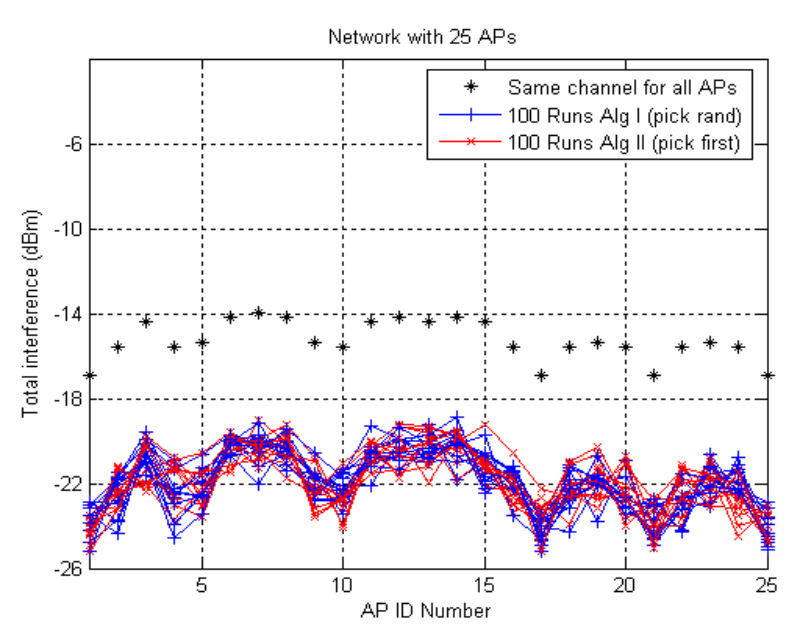

Fig. 4. Total Interference for AP 25 using algorithm I (pick rand) and II (pick first) comparing with same channel assignment. The different lines correspond to different initial conditions (i.e., initial AP channel numbers).

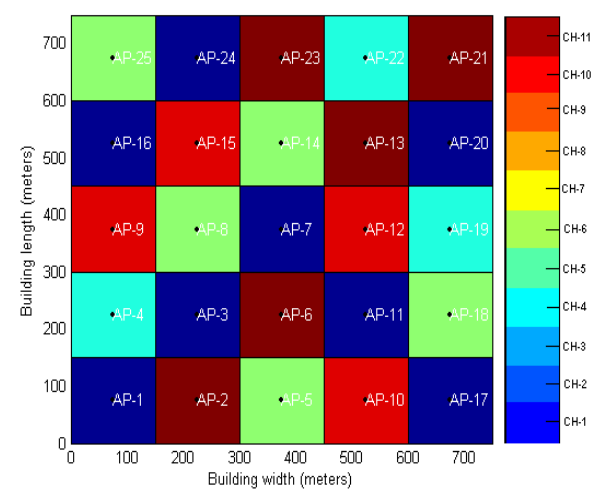

Fig. 5. Dynamic channel assignment map for WLAN with 25 APs.

ment and simulate our algorithm using two versions (I: pick rand and II: pick first). The simulations were carried out with service areas consisting of 4, 9, 16, and $25 \mathrm{APs}$, calculating the interference caused on an individual AP while minimizing the interference by dynamically assigning channels. We compared our results from algorithms I and II with the default settings of having all APs use the same channels. Our results showed an improvement by a factor of 4 by lowering the total interference on an AP by $6 \mathrm{dBm}$ on average over the default settings, which leads to significantly higher network throughput. In all the simulations we ran, the iterative approach converged to a unique solution in under 50 iterations (which only took a few seconds).

\section{REFERENCES}

[1] Y. Lee, K. Kim, and Y. Choi, "Optimization of AP placement and channel assignment in wireless LANs," LCN 2002. 27th Annual IEEE Conference on Local Computer Networks, pp. 831-836, November 2002.

[2] A. Hills and R. Friday, "Radio resource management in wireless LANs," IEEE Radio Communications, pp. S9-S14, December 2004.
TABLE IV

INTERFERENCE CALCULATED AT THE APS AFTER RUNNING DYNAMIC CHANNEL ASSIGNMENT ALGORITHM FOR WLAN WITH 25 APs.

\begin{tabular}{|c|c|c|}
\hline AP ID & CHANNEL $F_{i}$ & INTERFERENCE $(\mathrm{dBm})$ \\
\hline \hline$A P_{1}$ & 1 & -22.6745 \\
\hline$A P_{2}$ & 11 & -22.6418 \\
\hline$A P_{3}$ & 1 & -20.5696 \\
\hline$A P_{4}$ & 5 & -21.8568 \\
\hline$A P_{5}$ & 6 & -22.9029 \\
\hline$A P_{6}$ & 11 & -20.4941 \\
\hline$A P_{7}$ & 1 & -20.5552 \\
\hline$A P_{8}$ & 6 & -20.3750 \\
\hline$A P_{9}$ & 10 & -21.6051 \\
\hline$A P_{10}$ & 10 & -21.6470 \\
\hline$A P_{11}$ & 1 & -20.8541 \\
\hline$A P_{12}$ & 10 & -19.5659 \\
\hline$A P_{13}$ & 11 & -19.6376 \\
\hline$A P_{14}$ & 6 & -20.6079 \\
\hline$A P_{15}$ & 10 & -20.0170 \\
\hline$A P_{16}$ & 1 & -22.4759 \\
\hline$A P_{17}$ & 1 & -23.3011 \\
\hline$A P_{18}$ & 6 & -21.4209 \\
\hline$A P_{19}$ & 5 & -20.7232 \\
\hline$A P_{20}$ & 1 & -23.1058 \\
\hline$A P_{21}$ & 11 & -22.7518 \\
\hline$A P_{22}$ & 5 & -22.5916 \\
\hline$A P_{23}$ & 11 & -21.4080 \\
\hline$A P_{24}$ & 1 & -22.8317 \\
\hline$A P_{25}$ & 6 & -24.0713 \\
\hline \hline & & \\
\hline \hline
\end{tabular}

[3] J. Park, S. Park, P. Cho, and K. Cho, "Analysis of spectrum channel assignment for IEEE $802.11 \mathrm{~b}$ wireless lan," The 5th International Symposium on Wireless Personal Multimedia Communications, vol. 3, pp. 1073-1077, October 2002.

[4] A. Hills and J. Schlegel, "Rollabout: A wireless design tool," IEEE Commun. Mag., vol. 42, pp. 132-138, February 2004.

[5] A. Mishra, S. Banerjee, and W. Arbaugh, "Weighted coloring based channel assignment for WLANs," ACM SIGMOBILE Mobile Computing and Communications Review, vol. 9, pp. 19-31, 2005.

[6] IEEE, "Wireless LAN medium access control (MAC) and physical layer (PHY) specifications - Amendment 2: Higher speed physical layer (PHY) extension in the $2.4 \mathrm{GHz}$ band," IEEE Standard 802.11b, 1999.

[7] —_, "Wireless LAN medium access control (MAC) and physical layer (PHY) specifications - Amendment 4: Further higher-speed physical layer extension in the $2.4 \mathrm{GHz}$ band," IEEE Standard 802.11g, 2003.

[8] F. Kelly, "Blocking probabilities in large circuit switched networks," Advances in Applied Probability, vol. 18, pp. 473-505, 1986.

[9] R. Akl and S. Park, "Optimal access point selection and traffic allocation in IEEE 802.11 networks," Proceedings of WMSCI 04: 9th World Multiconference on Systemics, Cybernetics, and Informatics: Communication and Network Systems, Technologies and Applications, vol. 8, pp. 75-79, July 2005 . 